\title{
Detection of Coronary Artery Diseases: Comparative Study of Multidetector Computed Tomography Angiogram (64 slice scanner) and Conventional Angiogram
}

\author{
M Shirin'1, SA Azad ${ }^{1}$, M Rahman², S Dina ${ }^{2}$, E Karim¹, A Hossain ${ }^{3}$, M Ullah $^{4}$ \\ ${ }^{1}$ Department of Radiology and Imaging, Bangabandhu Sheikh Mujib Medical University, ${ }^{2}$ Department \\ of Radiology and Imaging, National Institute of Cardiovascular Diseases, ${ }^{3}$ Department of Radiology \\ and Imaging, Dhaka Medical College Hospital, ${ }^{4}$ Department of Cardiology, National Institute of
}

Cardiovascular Diseases

Key Words : Coronary artery

disease,

$M D C T$,

Angiogram

\begin{abstract}
:
Background: Multislice computed tomography (MDCT) is a promising noninvasive method of detecting coronary artery disease. It is not yet being widely used in Bangladesh. We aimed to assess the diagnostic accuracy of the of 64 slice $C T$ in detecting CAD with reference to conventional coronary angiography.
\end{abstract}

Methods: It was cross sectional comparative study conducted in National Institute of Cardiovascular Diseases and Bangabandhu Sheikh Mujib Medical University. Total 80 patients were included in the study who underwent MDCT and coronary angiogram.

Results: The sensitivity, specificity, positive predictive value, negative predictive value and accuracy or efficacy for single vessel disease were $87.5 \%, 96.4 \%, 91.3 \%, 94.7 \%$ and $93.75 \%$ respectively. The sensitivity, specificity, positive predictive value, negative predictive value and accuracy or efficacy for double vessel disease were $84.0 \%, 94.5 \%, 87.5 \%, 92.85 \%$ and $91.25 \%$ respectively. The sensitivity, specificity, positive predictive value, negative predictive value and accuracy or efficacy for triple vessel disease were $69.5 \%, 91.22 \%, 76.19 \%, 88.13 \%$ and $85.0 \%$ respectively.

Conclusion: Coronary angiography is a promising technology that already appears to have high performance to assess patient base, vessel base and segment base sensitivity, specificity, positive predictive value, negative predictive value and accuracy in detecting coronary artery stenosis.

(Cardiovasc. j. 2012; 4(2): 120-126)

\section{Introduction}

Coronary artery disease (CAD) and its consequences remain a leading cause of morbidity and mortality among most age groups in the USA and most Western countries of key words. ${ }^{1}$ It is predicted that the burden of cardio-vascular disease will continue to increase over the next 20 years (coronary artery disease, MDCT, CT Angiogram). Ischemic heart disease is responsible for $20 \%$ of all deaths of Bangladeshi people, as other South Asians, have high susceptibility to ischemic heart disease (IHD). The prevalence of IHD in Bangladesh is $3.4 \%$ in general, $4.6 \%$ in men and, $2.7 \%$ in women. It appears that IHD is an important problem even in rural population of Bangladesh.

Owing to its superior spatial and temporal resolution conventional invasive coronary angiography (CICA) remains the gold standard for the diagnosis of coronary artery diseases (CAD). However, it is an invasive and expensive procedure with preoperative preparation and per and post operative complications. It is associated with adverse events like vascular complications (1.6\%), arrythmia (0.3\%), stroke $(0.1 \%)$ and myocardial infarction $(<0.05 \%)$ and death $(0.12 \%){ }^{2,4}$ So, to reduce patient discomfort and procedural risk there are look out for an accurate, noninvasive alternative. ${ }^{4}$

The recent development of electrocardiographically gated multi-detector row computed tomography now almost replacing the conventional coronary angiography as a highly accurate diagnostic procedure. ${ }^{5}$ This also significantly reduces circumstancial factors and per and post operative complications related to conventional angiography.

Several key advances have facilitated the development of cardiac CT imaging like - 
- Spatial resolution in submillimeter level of permits the visualization of small cardiac structures and even side branches which allows not only the accurate visualization of coronary stenosis but also allows for the characterization of atherosclerotic plaques and the identification of plaque components which could lead to thromboembolic manifestation. Where as conventional angiogram fails to provide information on type and component of the plaque. $^{2}$

- Faster gantry rotation with improved temporal resolution and retrospective electrocardiogram (ECG) gating have reduced motion artifacts and improved image quality.

- Image quality is also improved by reducing the scan duration by having its multidetector and helical scan technology, and entire heart can be imaged in a single breathhold. ${ }^{4}$

(a) Provide maximum useful information by using MIP/MPR/VRT technique and provide better delineation of stenosis at the origin of the right and left coronary artery.

(b) Another advantage of MDCT angiography over coronary angiography lies in the fact that MDCT allows for the characterization of atherosclerotic plaques and the identification of plaque components. Conventional invasive coronary angiography is restricted to visualizing the luminal stenosis, but fails to provide information about the type of plaques or identify vulnerable plaques that could lead to thrombosis or myocardial infarction. Therefore, MDCT angiography has been increasingly used for the detection of coronary stenosis, the evaluation of coronary plaques and the prediction of disease progression. ${ }^{2}$

- This non-invasive diagnostic modality also allows to see the patency of stent and patency of the graft both arterial and venous. This also can evaluate anomalous origin of the coronary arteries and can detect congenital heart disease accurately.

- Functional assessment (ejection fraction) of ventricles can be done.

- It acts as a one stop shop analysis where we can see the cardiac structures (coronary artery valve, ventricular function, plaque morphology) as well as pulmonary vasculature in the single procedure.

Multislice computed tomography is a promising noninvasive method of detecting coronary artery disease. Most data regarding the use of MSCT in detecting coronary artery disease have been obtained from journals published abroad. It is not yet being widely used in Bangladesh. Further more accuracy of MSCT has not been tested in our population. Based on this context the current study was conceptualized with the hypotheis that, multislice coronary angiogram using 64 slice scanner is a useful diagnostic modality for detecting $\mathrm{CAD}$. We aimed to assess the diagnostic accuracy of 64-slice $\mathrm{CT}$ in detecting $\mathrm{CAD}$ with reference to conventional coronary angiography, primarily towards recommending the use of 64 -slice CT angiogram in diagnosis of CAD.

\section{Methods}

Study design

This study was a cross-sectional comparative study. Place of Study: a) Department of Radiology and Imaging, Institute of Cardiovascular Disease and Hospital (NICVD) and Department of Cardiology, Institute of Cardiovascular Disease and Hospital (NICVD) and Bangabandhu Sheikh Mujib Medical University, Dhaka.

Duration of study

From January 2009 to June 2010.

\section{Sample size determination}

Sample size was determined by the formula Sample $(\mathrm{n})=\mathrm{z}^{2} \mathrm{pq} / \mathrm{d}^{2}$.

Where, $z=1.96$ (at 95\% confidence level), p= $0.046=0.05$ (The prevalence of IHD is $4.6 \%$, Zaman et al 2007), $\mathrm{q}=1-\mathrm{p}=0.95, \mathrm{~d}=0.05$ (When acceptance of error is $5 \%$ )

so, $\mathrm{n}=1.96 \mathrm{X} 1.96 \mathrm{X} 0.05 \mathrm{X} .95 /(0.05)^{2}=72.9=73$.

Considering $10 \%$ drop out we took 80 (eighty) as sample size. A purposive and convenient sampling technique was done, described below.

\section{Study population and subjects}

Patients with suspected CAD who were referred to the Department of Radiology and Imaging in NICVD for evaluation of CAD during the study period were included in the study. During the study period a total of 200 patients were referred to the department of Radiology and Imaging to do CT angiogram. Adult patients of both sexes with 
suspected CAD (Atypical chest pain, Stable and unstable angina pectoris, regular heart rate, willing to go both for MDCT and invasive coronary angiography) were included. Patients with diagnosed case of CAD, with arrhythmia and refused to undergo catheter angiogram, known hypersensitivity to iodinated contrast material, renal insufficiency and severe congestive heart failure, and those who were unable to breath hold command were excluded. Among 200, catheter angiogram could not be done in 90 persons. Among remaining 110 persons who had undergone CT coronary angiogram all were also advised to undergo catheter angiogram for further evaluation. We could not collect the report of 30 patients among them who underwent catheter angiogram. Finally both CT and catheter angiogram reports were available in 80 cases for final analysis.

\section{Procedure}

Coronary CTA was obtained for all patients following the scan protocol. MDCT was performed and reported by expert radiologists. All eligible patients underwent invasive angiography via a femoral approach. All angiograms were evaluated by a team of expert cardiologists blind to MDCT results.

\section{Data analysis}

Sensitivity, specificity, positive predictive value (PPV) and negative predictive value (NPV) was calculated for MDCT using conventional CA as the gold standard. SPSS version 12.0 for windows statistical software was used for all data entry and statistical analysis.

\section{Ethical consideration}

Then written consent was taken from every patient. This protocol was submitted to the "Ethical Review Committee" of Bangabandhu Sheikh Mujib Medical University, Dhaka, Bangladesh.

\section{Results}

\section{Patient profile}

The mean $( \pm \mathrm{SD})$ age of the patients was $51.44( \pm 10.6)$ years with a range 31 to 75 years. Most of the patients were in age group 51-60 years (37.5\%) and $41-50$ years (32.5\%). 64 (80\%) patients were male and 16(20\%) patients were female. The male female ratio was $4: 1$. The mean $( \pm \mathrm{SD}) \mathrm{BMI}$ of the patients was 23.89 $( \pm 3.07)$ and the mean $( \pm$ SD) calcium score of the patients was $127.15( \pm 185.96)$. The mean $( \pm$ SD) heart rate during the MDCT scans was $73.25( \pm 10.26) \mathrm{bpm}$. Table 1 shows selected profile data of the study subjects.
Table-I

Profile of study subjects

\begin{tabular}{lcc}
\hline Age group & Frequency $(\mathrm{n}=80)$ & Percent \\
\hline 31-40 years & 12 & 15.0 \\
41-50 years & 26 & 32.5 \\
51-60 years & 30 & 37.5 \\
61-70 years & 9 & 11.3 \\
> 70 years & 3 & 3.8 \\
Sex & & \\
Male & 64 & 80.0 \\
Female & 16 & 20.0 \\
\hline
\end{tabular}

Image quality

The image quality was high in 53 of 80 (66.3\%), moderate in $22(27.5 \%)$ and poor in $5(6.3 \%)$ patients [Table 2].

Table-II

Image quality of MDCT angiogram

\begin{tabular}{lcc}
\hline Image quality & Frequency $(\mathrm{n}=80)$ & Percent \\
\hline High & 53 & 66.3 \\
Moderate & 22 & 27.5 \\
Poor & 5 & 6.3 \\
\hline
\end{tabular}

Table- 3 shows that CT angiogram could detect coronary artery disease in 68 (85\%) patients among 80 patients with chest pain. $12(15 \%)$ patients were found to be normal. Among the 68 patients 23 (28.75\%) had single vessel disease, $24(30.0 \%)$ had double vessel disease and 21(26.25\%) had triple vessel disease.

Table-III

CT finding of coronary disease

\begin{tabular}{lcc}
\hline CT Findings & Frequency & Percent \\
\hline Normal CT coronary & 12 & 15.00 \\
angiogram & & \\
Single vessel disease & 23 & 28.75 \\
Double vessel disease & 24 & 30.0 \\
Triple vessel disease & 21 & 26.25 \\
\hline
\end{tabular}

Analysis by involvement of segments of arteries In case of the RCA, the sensitivity and specificity of MDCT for the different segments are $92 \%$ and $92.7 \%$ for the proximal, $85.7 \%$ and $94.9 \%$ for the mid and $71.4 \%$ and $96.9 \%$ for the distal. In case of the LAD, the sensitivity and specificity of MDCT for the different segments are $100 \%$ and $100 \%$ for the left main, $89.6 \%$ and $198 \%$ for the proximal LAD, $78.1 \%$ and $91.7 \%$ for the mid $\mathrm{LAD}$ and $65 \%$ and $96.6 \%$ for the distal LAD [Table 4]. In case of the LCX, the sensitivity and specificity of MDCT for the different segments are $71.4 \%$ and $71.4 \%$ for the proximal, $72.7 \%$ and $91.3 \%$ for the distal [Table 5]. 
Table-IV

Diagnostic performance of MDCT CA for LAD artery disease

\begin{tabular}{lccccc}
\hline Left coronary artery & Sensitivity & Specificity & $\begin{array}{c}\text { Positive } \\
\text { predictive value }\end{array}$ & $\begin{array}{c}\text { Negative } \\
\text { predictive value }\end{array}$ & $\begin{array}{c}\text { Accuracy or } \\
\text { efficacy }\end{array}$ \\
\hline Left main & $100 \%$ & $100 \%$ & $100 \%$ & $100 \%$ & $100 \%$ \\
Proximal LAD & $89.6 \%$ & $98.0 \%$ & $96.3 \%$ & $94.3 \%$ & $95 \%$ \\
Mid LAD & $78.1 \%$ & $91.7 \%$ & $86.2 \%$ & $86.3 \%$ & $86.2 \%$ \\
Distal LAD & $65 \%$ & $96.6 \%$ & $86.6 \%$ & $89.23 \%$ & $88.75 \%$ \\
\hline
\end{tabular}

Table-V

Diagnostic performance of MDCT CA for left circumflex artery disease

\begin{tabular}{lccccc}
\hline Left circumflex & Sensitivity & Specificity & $\begin{array}{c}\text { Positive } \\
\text { predictive value }\end{array}$ & $\begin{array}{c}\text { Negative } \\
\text { predictive value }\end{array}$ & $\begin{array}{c}\text { Accuracy or } \\
\text { efficacy }\end{array}$ \\
\hline Proximal segment & $71.4 \%$ & $71.4 \%$ & $83.3 \%$ & $90.3 \%$ & $88.75 \%$ \\
Distal segment & $72.7 \%$ & $91.3 \%$ & $76.2 \%$ & $89.8 \%$ & $86.3 \%$ \\
\hline
\end{tabular}

Analysis by number of arteries involved and per patient

The sensitivity, specificity, positive predictive value, negative predictive value and accuracy or efficacy for single vessel disease were $87.5 \%, 96.4 \%, 91.3 \%$, $94.7 \%$ and $93.75 \%$ respectively. The sensitivity, specificity, positive predictive value, negative predictive value and accuracy or efficacy for double vessel disease were $84.0 \%, 94.5 \%, 87.5 \%, 92.85 \%$ and $91.25 \%$ respectively. The sensitivity, specificity, positive predictive value, negative predictive value and accuracy or efficacy for triple vessel disease were $69.5 \%, 91.22 \%, 76.19 \%, 88.13 \%$ and $85.0 \%$ respectively. Among the 80 subject MDCT CA diagnosed 68 patients as positive. Out of these 68 positive patients conventional coronary angiogram diagnosed 65 of them as positive. Statistical analysis for performance of the test revealed the sensitivity, specificity, positive predictive value, negative predictive value and accuracy or efficacy for per patient analysis as, $97.01 \%, 76.9 \%, 95.5 \%, 83.33 \%$, 93.75\% respectively [Table 6 ]

Table-VI

Diagnostic performance of MDCT CA by vessel based and per patient analysis

\begin{tabular}{lccccc}
\hline Vessels & Sensitivity & Specificity & PPV & NPV & Accuracy/efficacy \\
\hline Single vessel disease & $87.5 \%$ & $96.4 \%$ & $91.3 \%$ & $94.7 \%$ & $93.75 \%$ \\
Double vessel disease & $84.0 \%$ & $94.5 \%$ & $87.5 \%$ & $92.85 \%$ & $91.25 \%$ \\
Triple vessel disease & $69.5 \%$ & $91.22 \%$ & $76.19 \%$ & $88.13 \%$ & $85.0 \%$ \\
Per patient analysis & $97.01 \%$ & $76.9 \%$ & $95.5 \%$ & $83.33 \%$ & $93.75 \%$ \\
\hline
\end{tabular}

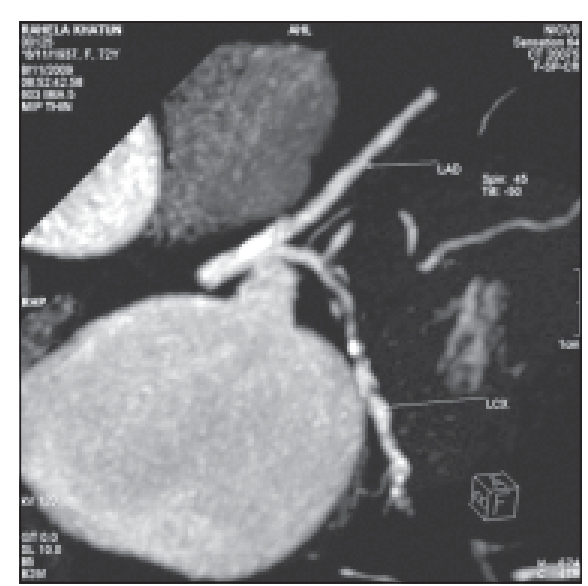

Fig.-1: 3D Axial image: single vessel disease showing stenosis at the LCX



Fig.-2: Double vessel disease (VRT image): stenosis in proximal segment of the $L A D \& L C X$ 


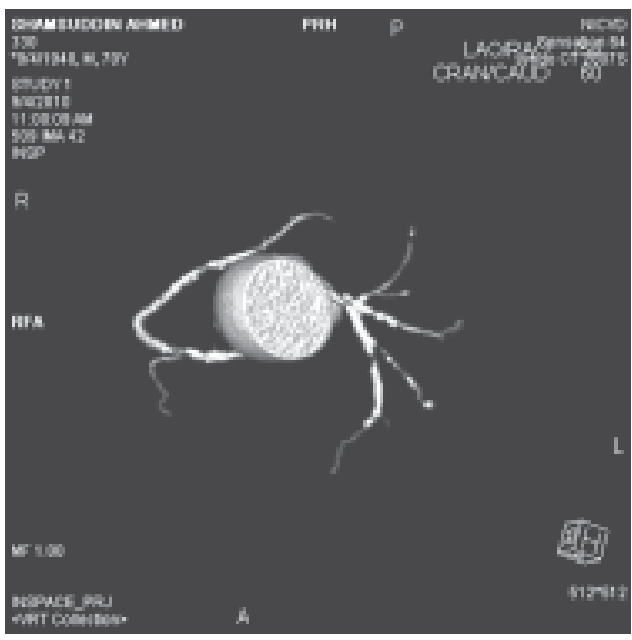

Fig.-3: Triple vessel disease (VRT image)

\section{Discussion:}

Our study found that mean $( \pm \mathrm{SD})$ age of the patients with chest pain was $51.44( \pm 10.6)$ years. This observation was supported by the findings. ${ }^{6}$ The authors recruited 716 individuals with mean $( \pm$ SD) of $55.2( \pm 15.2)$ years. The present study also revealed that the most of the sufferers are in age group $51-60$ years $(37.5 \%)$ and $41-50$ years (32.5\%). The male female ratio of the present study was 4:1. However other studies conducted by Shan et al and Becker et al found the male female ratio 2.1:1 and 1.5:1 which are not in agreement with our study findings. ${ }^{5}$ The difference may be due to the fact that IHD is more prevalent in men than female. In addition, in our hospitals, bed allocation for male patients is more than female patients. As the females are more conservative and ignored by the family they have tendency to hide their disease. The mean $( \pm \mathrm{SD}) \mathrm{BMI}$ of the patients was $23.89( \pm 3.07)$ with a range 19.2 to 35.04 and the chief complain were chest pain only $(63.8 \%)$, chest pain on exertion $(3,8 \%)$, with hypertension (17.5\%). Unstable angina was present in $3.8 \%$ patients and $7.5 \%$ had hypertension along with unstable angina. Nonspecific chest pain was present in $3.8 \%$ patients.

Our study had $66.3 \%$ CT angiogram with high, $27.5 \%$ with moderate and $6.3 \%$ with poor quality imaging. Our findings were in agreement with Nikolau et al. ${ }^{7}$ They also achieved $64 \%$ high, 30\% moderate and $6 \%$ poor quality imaging. The diagnostic performance of MDCT CA in comparison with CCA for the detection of significant CAS has been reported in multiple papers. In most of these studies, any lumen reduction greater than or equal to $50 \%$ was considered a significant coronary stenosis. Currently, 64-detector MDCT technology offers faster gantry rotation time: better temporal and spatial resolution and increased tube power (up to $1050 \mathrm{mAs}$ ) which is useful even in the patients with body mass index $>25$ to improve the diagnostic accuracy of the procedure and diminished the number of nonassessable segments.

Our study showed that MDCT CA diagnosed 68 (out of 80) patients as positive. Conventional coronary angiogram revealed 65 of them positive (stenosis $>50 \%$ ) and 3 found to be negative. On the other hand MDCT CA diagnosed 12 (out of 80) patients as negative. Conventional coronary angiogram revealed 2 of them positive and 10 found to be truly negative. Statistical analysis for performance of the test revealed the following results: sensitivity $97.01 \%$, specificity $76.9 \%$, positive predictive value $95.5 \%$, negative predictive value $83.33 \%$, accuracy or efficacy $93.75 \%$. These findings were supported by the observation of Leber and his associates, Nikolau et al, Selcoki et al. Raff et al. ${ }^{10}$ Leber et al found that the sensitivity was $95 \%$, specificity $90 \%$; positive predictive value $74 \%$, negative predictive value $98 \%$. They conducted the study among 90 patients. CT angiogram was done by dual X-ray source MDCT with high temporal resolution to rule out coronary stenosis. ${ }^{8}$

Nikolau et al (2006) found the per patient sensitivity $97 \%$, specificity $79 \%$; positive predictive value $86 \%$, negative predictive value $96 \% .{ }^{7}$ They conducted a study with 72 patients by MDCT. Selcoki et al (2010) found the per patient sensitivity $95.1 \%$, specificity $83.3 \%$; positive predictive value $96.7 \%$, negative predictive value $76.9 \%$. They conducted a study with 73 patients and examined by 64 -slice CT. Raff et al found that per patient specificity, sensitivity, and positive and negative predictive values for the presence of significant stenosis were $95 \%, 90 \%, 93 \%$, and $93 \%$, respectively. ${ }^{9}$ They studied 70 consecutive patients undergoing elective invasive coronary angiography to evaluate the diagnostic accuracy of multislice computed tomography (MDCT) coronary angiography using a 64-slice scanner.

Achenbach and colleagues have compared the diagnostic performance of MDCT CA using 16-and 64-detector CT systems, showing that at experienced centers and with dedicated data 
acquisition and analysis, sensitivities ranging from $83 \%$ to $99 \%$, specificities ranging from $93 \%$ to $99 \%$, and negative predictive values ranging from $95 \%$ to $100 \%$ can be achieved for the detection of relevant coronary artery stenosis. ${ }^{11,12}$ The present study revealed that the diagnostic performance in segment bases analysis of proximal segment of right coronary artery as follows: sensitivity $92 \%$, specificity $92.7 \%$, positive predictive value $85.18 \%$, negative predictive value $96.2 \%$ and an accuracy or efficacy $92.5 \%$. Our findings were supported by Nikolau et al and Selcoki et al. ${ }^{7,10}$ Nikolau et al found sensitivity $87 \%$, specificity $96 \%$, positive predictive value $74 \%$, negative predictive value $98 \%$, and accuracy or efficacy $95 \%$.

Selcoki et al found sensitivity $95.5 \%$, specificity $86.3 \%$, positive predictive value $75 \%$, negative predictive value $97.8 \% .^{10}$

The present study found that sensitivity, specificity, positive predictive value, negative predictive value and accuracy or efficacy of left main coronary artery was $100 \%$. Our findings were supported by Nikolau et al and Selcoki et al. ${ }^{7,10}$ Both the studies found that all values of sensitivity, specificity, positive predictive value, negative predictive value, and accuracy or efficacy were $100 \%$ for left main coronary artery.

The present study found that the diagnostic performance for the proximal part of left anterior descending coronary artery as follows was sensitivity $89.6 \%$, specificity $98 \%$, positive predictive value $96.3 \%$, negative predictive value $94.3 \%$, and accuracy or efficacy $95 \%$. Our findings were supported by Nikolau et al and Selcoki et al. ${ }^{7,10}$ Nikolau et al found sensitivity $78 \%$, specificity $92 \%$, positive predictive value $70 \%$, negative predictive value $95 \%$, accuracy or efficacy $90 \%{ }^{7}$ Selcoki et al found sensitivity $91.1 \%$, specificity $75 \%$, positive predictive value $85.4 \%$, negative predictive value $84.0 \%{ }^{10}$

Our study found that diagnostic performance for proximal segment of left circumflex coronary artery as follows: sensitivity $81.4 \%$, specificity $71.4 \%$, positive predictive value $83.33 \%$, negative predictive value $90.3 \%$, and accuracy or efficacy $88.75 \%$. Our findings were supported by Nikolau et al. and Selcoki. ${ }^{7,10}$ Nikolau et al found sensitivity $83 \%$, specificity $94 \%$, positive predictive value $71 \%$, negative predictive value $96 \%$, accuracy or efficacy $94 \% .^{7}$ Selcoki et al found sensitivity $65.4 \%$, specificity $95.7 \%$, positive predictive value $89.5 \%$, and negative predictive value $83.3 \% .^{10}$

Certain limitations of our study must be mentioned. The study was carried out in specialized tertiary level hospital which is situated in the capital city, so the results obtained from this study may not represent the whole population. As the study was carried out in a short period of time expected number of sample could not be collected resulting into a smaller sample size.

\section{Conclusion:}

Coronary angiography is a promising technology that already appears to have high performance to assess patient base, vessel base and segment base sensitivity, specificity, positive predictive value, negative predictive value and accuracy in detecting coronary artery stenosis. Patients who are unwilling to undergo an invasive process MDCT can serve as an excellent screening procedure which will reduce the load on invasive conventional procedure. Because of its high spatial resolution and ability to image the arterial lumen and wall, further technological development will likely make this an important non- invasive diagnostic tool for cardiovascular pathophysiology.

\section{References:}

1. Hoffmann U, Bamberg F, Chae CU, Nichols JH, Rogers IS, Seneviratne SK, et al. 'Coronary Computed Tomography Angiography For Early Triage of Patients with Acute Chest Pain - The Rule Out Myocardial Infarction Using Computer Assisted Tomography (ROMICAT) Trial'. J Am Coll Cardiol 2009; 53:16421650 .

2. Nieman K, Ooijen P; Rensing B, Oudkerk M n and de Feyter PJ. Four-Dimensional Cardiac Imaging with Multislice Computed Tomography. Circulation 2001;103:62.

3. Nieman K, Oudkerk M, Rensing BJ, van Ooijen P, Munne A, van Geuns RJ, de Feyter PJ. Coronary angiography with multi-slice computed tomography. Lancet 2001.; 24:599-603.

4. Sun Z and Ng KH. Multislice CT angiography in cardiac imaging. Part II: clinical applications in coronary artery disease. Singapore Med Journal 2010; 51: 282-286.

5. Chow BJW, Hoffmann U and Neimann K. Computed tomographic coronary angiography:An alternative to invasive coronary angiography. Can J Cardiol 2005; 21: 933-40. 
6. Zaman MM, Ahmed J, Choudhury SR, Numan SM, Parvin K, Islam MS. Prevalence of ischemic heart disease in a rural population of Bangladesh. Indian Heart J 2007; 59: 239-41.

7. Shan Y, Meng-su Z, Zhi-yong Z, Zhi-qing L, Jian-ying $\mathrm{M}$ and Gang C. Sixty-four-multi-detector computed tomography diagnosis of coronary artery anomalies in 66 patients. Chin Med J 2010; 123: 838-842.

8. Becker A, Leber AW, Becker C, von Ziegler F, Tittus J, Schroeder I et al. Predictive value of coronary calcifications for future cardiac events in asymptomatic patients with diabetes mellitus: A prospective study in 716 patients over 8 years. BMC Cardiovascular Disorders 2008; 8: 206.

9. Nikolaou K, Becker CR, Wintersperger BJ, Rist C, Trumm C, Leber A, Babaryka G, Reiser MF. Evaluating multislice computed tomography for imaging coronary atherosclerosis. Radiologe 2004; 44:130-9.

10. Nikolaou K, Knez A, Rist C, Bernd J. Wintersperger BJ, Leber A,et al. Accuracy of 64-MDCT in theDiagnosis of Ischemic Heart Disease. AJR 2006;187 :111-117.

11. Leber AW, Johnson T, Becker A, von Ziegler F, Tittus J, Nikolaou K et al. Diagnostic accuracy of dual-source multi-slice CTcoronary angiography in patients with an intermediate pretest likelihood for coronary artery disease. European Heart Journal 2007; 28: 2354-2360.

12. Raff GL, Gallagher MJ, O’Neill WW, Goldstein JA . Diagnostic accuracy of noninvasive coronary angiography using 64-slice spiral computed tomography. J Am Col Cardiol 2006; 46: 552-7.

13. Achenbach S, Giesler T, Ropers D, Ulzheimer S, Derlien $\mathrm{H}$, Christoph Schulte C et al. Detection of Coronary Artery Stenoses by Contrast-Enhanced, Retrospectively Electrocardiographi-cally- Gated, Multislice Spiral Computed Tomography. Circulation 2010;103: 25352538.

14. Haaga JR, Lanzieri CF, Gilkeson RC, Coronary artery diseas. In: Computer tomography and magnetic resonance Imaging of the whole body. Vol 2. $4^{\text {th }}$ edition. 2006:181-1201.

15. Selcoki Y, Caglar O, Kankilic MN, Akin K and Eryonucu B. Diagnostic accuracy of 64 slice computed totmography in patients with suspected or proven coronary artery disease. Turk Kardiyol Dern Arch-Arch Turk Soc CArdiol 2010; 38: 95-100. 